$\left.163\right|_{\text {LA-5126-MS }}$

th. 2426

INFORMAL REPORT

$r \mid 29173$

Magnet Alignment Study for the LAMPF Biomedical Pion Channel

scion limos of the University of California LOS ALAMOS, NEW MEXICO 87544 t

MASTER

ATOMIC ENERGY COMMISSION
CONTRACT W-74OS-ENG. 36

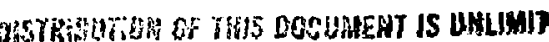


This report was prepared as an account of work sponsored by the United States Government. Neither the United States nor the United States Atomic Energy Commission, nor any of their employees, nor any of their contractors, subcontractors, or their employees, makes any warranty, express or implied, or assumes any legal liability or responsibility for the accuracy, completeness or usefulness of any information, apparatus, product or process disclosed, or represents that its ise would not infringe privately owned rights.

In the interest of prompt distribution, this LAMS report was not edited by the Technical Information staff.

Printed in the United States of America. Available from National Technical Information Service

U. S. Department of Commerce 5285 Port Royal Road Springfield, Virginia 22151

Price: Printed Copy $\$ 3.00$; Microfiche $\$ 0.95$ 
LA-5126-MS

Informal Report

UC-38

ISSUED: January 1973

\section{Magnet Alignment Study for the LAMPF Biomedical Pion Channel}

by

Richard L. Hutson

This work supported by the U.S. Atomic Energy Commission's Division of Biology and Medicine. 
MAGNET ALIGNMENT STUDY FOR THE

LAMPF BIOMEDICAL PION CHANNEL

by

Richard L. Hutson

\section{ABSTRACT}

Magnet alignment tolerances for the LAMPF Biomedical Pion Channel are specified and the effects of misalignment are discussed.

I. INTRODUCTION

A. Background

The LAMPF biomedical pion channel comprises eleven magnets, eight quadruple magnets and three bend magnets. Fig. I is a schematic elevation view of the channel.

To assure that the LAMPF biomedical pion channel will transport the maximum possible number of pions per unit time and so that it will focus the pien beam onto the volume to be irradiated with minimal centroid displacement and momentum spread, it is necessary to align the channel magnets within certain tolerances.

Alignment tolerances are specified in this report, and the effects of misalignment are discussed. B. Definitions

The descriptions given below are meant to clarify the meaning of terms used in the report, and to make slear the difference between measured quantities and ideal or defined quantities.

\section{Defined Quantities}

a. Channel Axis. This corresponds to the term, central trajectory, as used in reference 1 .

b. Target Center. The initial point of the channel. It is the intersection of the axis of the accelerator bean and the channel axis.

c. Bend plane. The vertical plane through the target center and containing the channel axis.

d. Pion Irradiation Position. The point on the channel axis corresponding to the nominal end of the channel. e. Magnet Entrance and Exit Points. Both an entrance and exit point are specified on the channel axis for each magnet. The magnet is aligned with respect to these points.

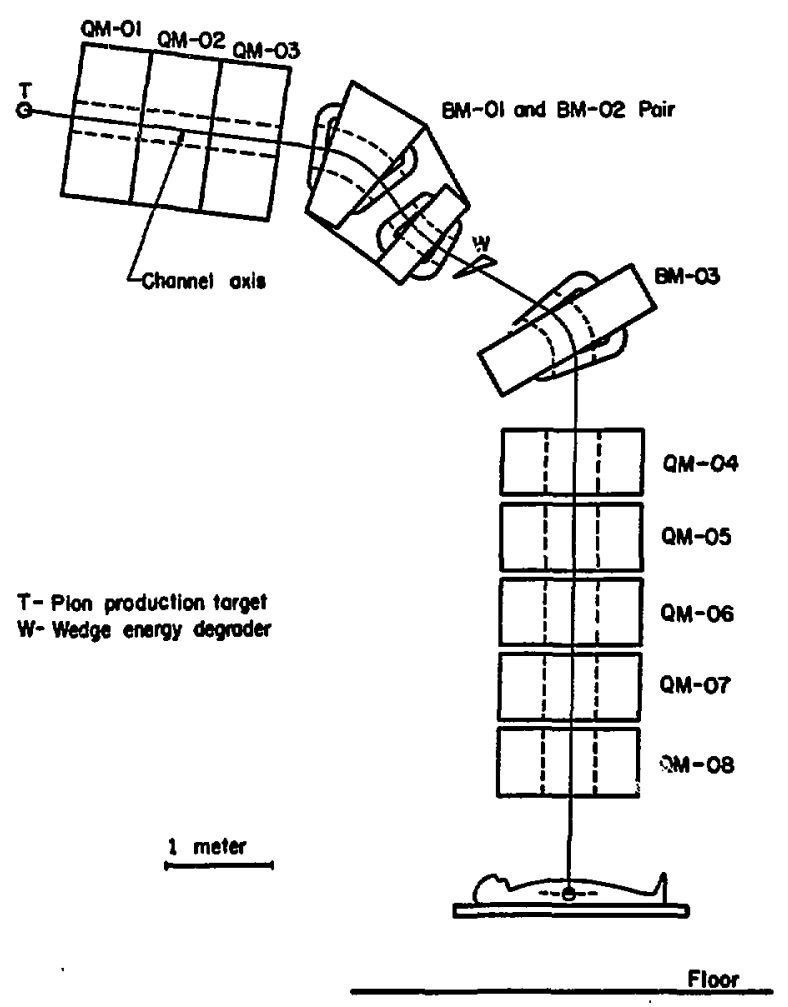

Fig. 1. The Los Alamos Meson Physics Facility Biomedical Pion Channel 


\section{Measured Quantities}

a. Magnet Reference Plane. A plane which is defined with respect to the poles of a magnet and which is aligned to be coinczdent with the bend plane. The reference plane for a quadrupole is that plane which bisects the angle between adjacent poles. The reference plane for a bend magnet is the median plane between the poles.

b. Magnet Entrance and Exit Axes. For a quadrupole the axes are the axis of the bore of the magnet. For a bend magnet, the magnet axes are determined by floating wire orbit measurements in the bend magnet reference plane. The magnet will be aligned so that the entrance and exit axes are coincident with the channel axis as it enters and leaves the magnet respectively.

\section{c. Magnet Entrance and Exit Reference}

Points. The positions of the quadrupole effective field boundaries along the magnet axis at the magnet entrance and exit respectively.

\section{MAGNET MISALIGNMENT EFFECTS}

There are three magnet misalignnent effects to be ccnsidered. They are:

(1) Beam centroid shift at the end of the cirannel.

(2) Beam centroid shift within the channel magnets. The magnets or beam pipe will scrape off beam if the shift is excessive.

(3) Beam centroid shift at the momentum dispersion plane. A wedge energy degrader is placed at the dispersion plane in such a way to degrade all pions to approximately the same energy. The effect of a centroid shift here is $t$ r change the mean energy of the pion beam leaving the wedge

Alignment tolerances will be stated for each of the eleven magnets in the channel. The cumulative effects of misalignments of these magnitudes on the beam centroid shifts will be summarized in Section VI.

\section{GENERAL DISCUSSION OF THE MAGNET ALIGNMENT PROCEDURE}

The LAMPF biomedical pion channel comprises eight quadrupole magnets and three bend magnets. The channel axis lies in a vertical plane, referred to as the bend plane, that passes through both the target center and the pion irradiation positon. All eight quadrupoles are positioned with their axis in the bend $\mathrm{p} 1$ ane and the coincident with the channel axis.
The reference planes of the bend magnets 1 ie in the bend plane.

Each quadrupole magnet or set of magnets, which is installed as a unit, will have two reference points and a reference plane through these points. Alignment will be with respect to the channel axis and the bend plane. The first quadrupole reference point is the position of the effective field boundary on the quadrupole axis at the entrance to the rnagnet. The second reference point is the corresponding point at the exit of the magnet. A quadrupole reference plane contains the quadrupole axis and bisects the angle between adjacent pole tips. There are two such planes in each quadrupole. The one referred to in this report is the one which is to be positioned coincident with the bend plane.

Each bend magnet or set of magnets will have two reference points and a reference plane through these points. The bend magnet median plane defines the reference $p l$ ane and the reference points are the positions of the effective field boundaries on the bend magnet entrance and exit trajectories.

Magnet reference points are to be aligned within tolerances along three mutually perpendicular axes. The first axis is referred to as the $x$ axis. It lies within the bend plane and at right angles to the channel axis at the position of the reference point that is being aligned. The $y$ axis is perpendicular to the bend plane at the point of interest. The $z$ axis coincides with the channel axis.

\section{BASIC DATA}

\section{A. Introductory Remarks}

Misalignment of each magnet or set of magnets was studied separately using the align option calculation that is a part of the beam optics code, TRANSPORT. 1

It was necessary to choose some particular channel tuning in order to study magnet misalignment effects. The tuning chosen was one that gives a noninal pion beam size at the irradiation position of $15 \mathrm{~cm}$ in the bend plane and $1.5 \mathrm{~cm}$ in the transverse plane. It was assumed that this tuning would result in misalignment effects which were representative of most useful tuning configurations. The TRANSPORT $^{1}$ code listing for the channel elements in this tuning configuration is given in Table $I$. 


\section{TABLE I}

LISTING OF TRANSPORT ${ }^{\text {a }}$ DECK SETUP USED FOR MISALIGNMENT STUDIES

Card Remarks

WHOLE CH FOR ALIGN RUNS

0

1. 2.5 150. 1. 50. 0. 0. .2025 BEAM

16. $7 . .45 \mathrm{~K} 1$

16. 8. $2.8 \mathrm{~K} 2$

$3.0 .50 \mathrm{Dl}$

5. $.524 .315 \quad 14 . Q 1$

3. .15 D

5. $.52-3.25014 . Q 2$

3. .15 D

5. $.52 .217 \quad 14 . \mathrm{Q3}$

3. .5 D2

16.05 .07 .62 GAP

2.00 .0 PFR 1

$24.0 .6283245,0.0$ B1

2.00 .0 PFR2

3.0 .4686 D5

20.0180 . ROT 1

16. 5. 5.08 GAP

2.00 .0 PFR3

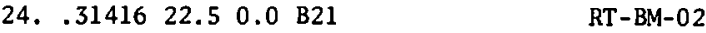

2.00 .0 PFR4

20. -180 . ROT2

3. $.4 \mathrm{D} 6$

3. $.8514 \mathrm{D} 7$

16. 5. 8.89 GAP

16. $7 . .4$ FRIN1

16. 8. 4.4 FRIN2

2. $19.703 \mathrm{Rl}$

24. .4356 60. 0. B3 RT-BM-03

2. $19.703 \mathrm{R} 2$

3. 1. 08

$\begin{array}{lllll}5.00 & .46 \quad 2.907 & 17.78 \quad Q 4\end{array}$

3. $.38 \mathrm{D}$

$\begin{array}{lllll}5.00 & .46 & -3.884 & 17.78 & Q 5\end{array}$

3. $.38 \mathrm{D}$

$5.00 .46-1.123 \quad 17.78$ Q6

RT-QM-01

RT-QM-02

3. $.38 \mathrm{D}$

$5.00 .46 \quad 4.445 \quad 17.78 \quad Q 7$

3. $.38 \mathrm{D}$

$\begin{array}{llll}5.00 & .46 & -5.649 & 17.78 \quad \mathrm{Q} 8\end{array}$

3. $.9 \mathrm{D} 9$

$\overline{a_{\text {See }} \text { reference } 1 .}$

Since the biomedical pion channel was designed to have flexibility in shaping the pion beam so that actual experience with using the channel can be used to arrive at optimum tuning for specific experiments, it is not possible to foresee all possible tunings and the corresponding misalignment effects in each case.

B. Misalignment Effects at the End of the Channel

Table II lists the effects of six different misalignments for each of the eleven channel magnets and for the entrance quadrupole triplet as a unit.
Each magnet was misaligned by $0,1 \mathrm{~cm}$ along the $x, y$, and $z$ axes separate $y, y$, the axes being defined at the entrance reference point of the magnet. Then the magnet was misaligned by rotating by $1.0 \mathrm{mr}$ around each axis siparately. The resulting beam centroid shifts of the spatial distributions ( $x$ and $y$ ) and the angular distributions ( $\theta$ and $\phi)$ at the end of the channel are tabuIated.

\section{Misaligninent Effects at the Wedge Energy De-} grader

The remarks made in Section IV.B. about misalignment effects at the end of the channel also apply to misalignment effects at the wedge energy degrader. In this case only the first three quadrupoles and the firșt two bend magnets have effect. Table III lists tine effects.

D. Misalignment Effects Within the Channel Magnets

In order to get a conservative estimate of the beam centroid shifts within the channel due to magnet misalignment, each element was misnligned and the maximum observed centroid shift was used as a measure of the typical centroid shift. These typical centroid shifts corresponding to the various misalignments are tabulated in Table IV. Using these maximum observed centroid shifts to calculate the cumulative effect of the misalignment of all the magnets will give an estimate significantly greater than woulc be expected at any one point in the channel. For this reason, the estimates are conscrvative for the purposes of evaluating the effects of misalignment.

\section{ALIGMMENT TOLERANCES}

\section{A. Introductory Remarks}

All alignment tolerance limits are stated in the form, \pm a cm. It is assumed that these limits represent the worst case misalignment and that there is uniform probability of any misalignment between the limits.

B. Alignment of the Entrance Quadrupole Triplet (RT-QM-01) through RT-QM-03)

The entrance triplet comprising the first three quadrupoles in the channel are assembled as a unit and will be aligned as a unit. The internal alignment tolerances specified for the assembly of the triplet are precise enough so that beam centroid shifts arising from the internal misalignments are small in comparison to the centroid shifts arising from misalignment of the triplet as a unit. 
TABLE II

CENTROID SHIFTS AT THE END OF THE CHANNEL DUE TO MAGNET MISALIGNMENTS

\begin{tabular}{|c|c|c|c|c|c|}
\hline \multirow{3}{*}{$\begin{array}{l}\text { Misaligned } \\
\text { Element } \\
\end{array}$} & \multirow{3}{*}{$\begin{array}{c}\text { Misalignment } \\
\text { Type } \\
\end{array}$} & \multicolumn{4}{|c|}{ Centroid Shifts $^{a}$} \\
\hline & & $x$ & $\theta$ & $y$ & $\phi$ \\
\hline & & $(\mathrm{cm})$ & $(m r)$ & $\underline{(\mathrm{cm})}$ & $(\mathrm{mr})$ \\
\hline \multirow[t]{6}{*}{ RT -QM-01 } & 1 & 0.522 & 3.890 & - & - \\
\hline & 2 & - & - & 0.03089 & 1.255 \\
\hline & 3 & - & - & -0.09981 & -3.609 \\
\hline & 4 & 0.1356 & 1.032 & - & - \\
\hline & 5 & - & - & - & - \\
\hline & 6 & - & - & - & - \\
\hline \multirow[t]{6}{*}{$R T-Q M-02$} & 1 & $-0.190 v$ & -1.937 & - & $\overline{-}$ \\
\hline & 2 & - & - & -0.05652 & -2.874 \\
\hline & 3 & - & - & 0.2090 & 10.51 \\
\hline & 4 & -0.04327 & -0.4800 & - & - \\
\hline & 5 & - & - & - & - \\
\hline & 6 & - & - & - & - \\
\hline \multirow[t]{6}{*}{$\mathrm{RT}-Q M-03$} & 1 & 0.005038 & 0.1352 & - & - \\
\hline & 2 & - & - & 0.002972 & 0.1672 \\
\hline & 3 & - & - & -0.01194 & -0.6608 \\
\hline & 4 & 0.001100 & 0.03586 & - & - \\
\hline & 5 & - & - & - & - \\
\hline & 6 & - & - & - & - \\
\hline \multirow[t]{6}{*}{ Triplet ${ }^{c}$} & 1 & 0.3371 & 2.088 & - & - \\
\hline & 2 & - & - & -0.1467 & -7.605 \\
\hline & 3 & - & - & 0.09726 & 6.237 \\
\hline & 4 & -0.02751 & -0.5293 & - & - \\
\hline & 5 & - & - & - & - \\
\hline & 6 & - & - & - & - \\
\hline \multirow[t]{6}{*}{ RT-BM-01 } & 1 & -0.05165 & 0.9011 & - & - \\
\hline & 2 & - & - & 0.01759 & 1.419 \\
\hline & 3 & - & - & -0.01176 & -0.8890 \\
\hline & 4 & -0.01711 & 0.2986 & - & - \\
\hline & 5 & -0.02860 & -0.7111 & - & - \\
\hline & 6 & - & - & -0.03804 & 2.840 \\
\hline \multirow[t]{6}{*}{ RT-BM-02 } & 1 & -0.04012 & 0.09502 & - & - \\
\hline & 2 & - & - & 0.002120 & 0.3488 \\
\hline & 3 & - & - & -0.003412 & -0.5116 \\
\hline & 4 & -0.006385 & 0.01512 & - & - \\
\hline & 5 & -0.0001337 & -0.4559 & - & - \\
\hline & 6 & - & - & 0.009069 & 1.354 \\
\hline \multirow[t]{6}{*}{ RT-BM-03 } & 1 & -0.04043 & -1.325 & - & - \\
\hline & 2 & - & - & -0.006663 & 1.050 \\
\hline & 3 & - & - & -0.01964 & 3.040 \\
\hline & 4 & -0.01299 & -0.2803 & - & - \\
\hline & 5 & -0.06942 & 0.2318 & - & - \\
\hline & 6 & - & - & -0.01990 & 3.069 \\
\hline \multirow[t]{6}{*}{ RT-QM-04 } & 1 & 0.03164 & -1.168 & - & - \\
\hline & 2 & - & - & -0.01045 & -0.3129 \\
\hline & 3 & - & - & 0.04298 & 1.071 \\
\hline & 4 & 0.008608 & -0.2510 & - & - \\
\hline & 5 & - & - & - & - \\
\hline & 6 & - & - & - & - \\
\hline \multirow[t]{6}{*}{ RT $-Q M-05$} & 1 & -0.1192 & -0.09817 & - & - \\
\hline & 2 & - & - & 0.02404 & 1.359 \\
\hline & 3 & - & - & -0.1046 & -5.757 \\
\hline & 4 & -0.03007 & -0.05903 & - & - \\
\hline & 5 & - & - & - & - \\
\hline & 6 & - & - & - & - \\
\hline \multirow[t]{6}{*}{ RT-QM-06 } & 1 & -0.09327 & -0.5879 & - & - \\
\hline & 2 & - & - & 0.002742 & 0.2628 \\
\hline & 3 & - & - & -0.01394 & -1.219 \\
\hline & 4 & -0.02305 & -0.1488 & - & - \\
\hline & 5 & - & - & - & - \\
\hline & 6 & - & - & - & - \\
\hline
\end{tabular}


TABLE II

(continued)

\begin{tabular}{|c|c|c|c|c|c|}
\hline \multirow{3}{*}{$\begin{array}{c}\text { Misaligned } \\
\text { Element }\end{array}$} & \multirow{3}{*}{$\begin{array}{c}\text { Misalignment } \\
\text { Type } \\
\end{array}$} & \multicolumn{4}{|c|}{ Centroid Shifts ${ }^{a}$} \\
\hline & & $x$ & $\theta$ & $y$ & $\phi$ \\
\hline & & $(\mathrm{cm})$ & $(m r)$ & $(\mathrm{cm})$ & $(\mathrm{mr})$ \\
\hline \multirow[t]{6}{*}{$\mathrm{R}^{\prime} \Gamma-\mathrm{QM}-07$} & 1 & 0.6437 & 4.663 & - & - \\
\hline & 2 & - & - & 0.01230 & -0.1765 \\
\hline & 3 & - & - & -0.04276 & 1.052 \\
\hline & 4 & 0.1461 & 1.066 & - & - \\
\hline & 5 & - & - & - & - \\
\hline & 6 & - & - & - & - \\
\hline \multirow[t]{6}{*}{ RT-QM-08 } & 1 & -0.2827 & -2.541 & - & - \\
\hline & 2 & - & - & -0.04845 & -0.4577 \\
\hline & 3 & - & - & 0.2098 & 1.822 \\
\hline & 4 & -0.05665 & -0.5403 & - & - \\
\hline & 5 & -. & - & - & - \\
\hline & 6 & - & - & - & - \\
\hline
\end{tabular}

\footnotetext{
on the $x-z$ plane and the $y-z$ planes respectively.

$b_{\text {Misalignment type }}$

- lateral misalignment in $x$ direction

- angular misaligrment around $x$ axis

- lateral misalignnent in y direction

- angular misalignment around y axis

- lateral misalignment in $z$ direction

- angular inisalignment around $z$ axis
}

$a_{\theta}$ and $\phi$ denote the angles between the pion trajectories and the channel axis ( $z$ axis) as projected

The lateral misalignments were all $0,1 \mathrm{~cm}$.

The angular misalignments were al $1.0 \mathrm{mr}$.

${ }^{c}$ Combination of RT-QM-01, RT-OM-02, and RT-QM-03.

Both the entrance and exit triplet reference points are to be positioned on the channel axis to within \pm 0.050 in. in both the $x$ and $y$ directions. The triplet reference plane is to be positioned to within \pm 0.050 in. of the bend p1ane at all points within the bore diameter of the triplet. Along the channel axis the triplet entrance reference point is to be positioned at $0.500 \mathrm{~m} \pm 0.050 \mathrm{in}$. from the target center,

C. Alignment of the First Two Bend Magnets (RT-BM-01 and RT-BM-02)

1. Definition of the Channe1 Axis for Aligning the Magnet Pair. The first two bend magnets have independent yokes, but are assembled as a unit with a pair of steel side plates to which both magnets are attached.

Although the positions of the effective field boundaries of the magnets were calculated as closely as possible, it is difficult to predict the precise effect of the combined fringe fields of the two magnets in the space between them. For this reason, it is proposed that reference points for aligning the magnet pair be established by making floating wire orbit measurements to define both entrance and exit axes for the pair. Entrance and exit reference points will then be defined to be on these axes at the magnet effective field boundaries at the encrance to the first bend magnet and at the exit of the second bend magnet respectively. The common liadian plane of the two bend magnets of the pair will be the reference plane for the pair. The measured configuration of the magnet pair entrance and exit axes and reference points will be used to define the channel axis in the region of the bend magnet pair. To do this project the measured configuration of the magnet pair axes and reference points onto the chame 1 bend plane in such a way that the entrance axis of the magnet pair is coincident with the first straight section of the channel axis and the entrance reference point is $2.860 \mathrm{in}$. from the target center. The channel axis in the region following the bend magnet pair will then be defined as that line coincident with the magnet pair exit axis and the magnet pair exit point will be defined to be coincident with the exit reference point. This is illustrated in Fig. 2

2. Alignment. The magnet pair entrance reference point is to be positioned along the first straight section of the channel axis at $2.860 \mathrm{~m} \pm 0.050 \mathrm{in}$. 
TABLE II I ${ }^{a}$

CENTROID SHIFTS AT THE WEDGE ENERGY DEGRADER DUE TO MAGNET MISALIGNMENTS

\begin{tabular}{|c|c|}
\hline $\begin{array}{c}\text { Misai igned } \\
\text { Element } \\
\end{array}$ & $\begin{array}{c}\text { Mi salignment } \\
\text { Type } \\
\end{array}$ \\
\hline$R T-Q M-01$ & $\begin{array}{l}1 \\
2 \\
3 \\
4 \\
5 \\
6\end{array}$ \\
\hline$R T-Q M-02$ & $\begin{array}{l}1 \\
2 \\
3 \\
4 \\
5 \\
6\end{array}$ \\
\hline RT $-Q M-03$ & $\begin{array}{l}1 \\
2 \\
3 \\
4 \\
5 \\
6\end{array}$ \\
\hline Triplet & $\begin{array}{l}1 \\
2 \\
3 \\
4 \\
5 \\
6\end{array}$ \\
\hline $\mathrm{RT}-\mathrm{BM}-01$ & $\begin{array}{l}1 \\
2 \\
3 \\
4 \\
5 \\
6\end{array}$ \\
\hline $\mathrm{RT}-\mathrm{BM}-02$ & $\begin{array}{l}1 \\
2 \\
3 \\
4 \\
5 \\
6\end{array}$ \\
\hline
\end{tabular}

$x$
$\frac{(\mathrm{cm})}{0.1816}$
-
-
0.04899
-
-
-0.1098
-
-
-0.02830
-
-
0.009962
-
-
0.002707
-
-
0.08174
-
-
-0.03683
-
-
0.09006
-
-
0.02984
-0.5180
-
0.01913
-
-
0.003045
-0.03827
-
-

Centroid Shifts

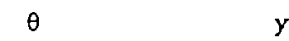

(mr) $\quad$ (cm)

$-6.213$

-

0.09947

$-0.3249$

$-1.612$

-

2.264

$-$

0.5139

$-$

$-0.05955$

$-$

$-$

$-$

$-0.1774$

0.6571

$-$

$-$

$-$

0.009203

$-0.03705$

0.01297

-

$-$

4.009

-

$0 . \overline{3258}$

$-$

0.6185

-

$0 . \overline{2049}$

0.3382

-

-

$-$

$-0.4594$

0.2951

-

-

0.

0.05107

$-0.03459$

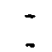

0.1122

0.4784

0.004745

$-0.008032$

0.07612

-

0.02140

$\phi$

(mr)

0.3569

$-1.027$

-

-

.8160

2.983

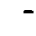

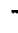

$-$

0.04745

$-0.1875$
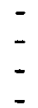

$-2.159$

1. 768

-

$-$

$-$

0.4020

$-0.2519$

-

0.8048

0.09859

$-0.1446$

-

0.3827

\footnotetext{
See Table II for explanation of terms.
}

from the target center. The entrance and exit reference points are to be positioned on the channel axis to within $\pm 0.050 \mathrm{in}$. in both the $x$ and $y$ directions. The magnet pair reference plane is to be within \pm 0.050 in. of the bend plane at all points between the magnet poles.

D. Alignment of the Third Bend Magnet (RT-BM-03)

1. Definition of the Channel Axis for Aligning the Magnet. Floating wire orbit measurements will be used, as was desiribed for the first two bend magnets, to establish entrance and exit reference points and axes for the third bend magnet (RT-BM-03). The measured configuration of the reference points and axes of RT-BM-03 will be used to define the channel axis in the region of RT-BM-03. The reference plane of $\mathrm{RT}-\mathrm{BM}-03$ is the median plane between the poles.

The measured RT-BM-03 configuration is projected onto the bend plane with the entrance axis coincident with the channel axis out of RT-BM- 02 and with the entrance reference point $1.251 \mathrm{~m}$ from the position of the RT-BM-02 exit point defined in the projection shown in Fig. 2, This is illustrated in Fig. 3. The channel axis coming out of RT-BM-03 is now defined to be coincident with the exit axis of RT-BM-03 and the exit point is defined to be 
TABLE IV ${ }^{\mathrm{a}}$

MAXIMUM CENTROID SHIFT WITHIN THE CHiLNNEL DUE TO MAGNET MISALIGMMENTS

\begin{tabular}{|c|c|c|c|c|c|c|c|}
\hline \multirow[b]{2}{*}{$\begin{array}{c}\text { Misaligned } \\
\text { Element }\end{array}$} & \multirow[b]{2}{*}{$\begin{array}{c}\text { Misalignment } \\
\text { Type } \\
\end{array}$} & \multicolumn{2}{|c|}{ Centroid Shifts } & \multirow[b]{2}{*}{$\begin{array}{c}\text { Misaligned } \\
\text { Element }\end{array}$} & \multirow[b]{2}{*}{$\begin{array}{c}\text { Misalignment } \\
\text { Type } \\
\end{array}$} & \multicolumn{2}{|c|}{ Centroid Shifts } \\
\hline & & $\begin{array}{c}x \\
(\mathrm{~cm})\end{array}$ & $\begin{array}{c}y \\
(\mathrm{~cm}) \\
\end{array}$ & & & $\begin{array}{l}x \\
(\mathrm{~cm}) \\
\end{array}$ & $\begin{array}{l}y \\
(\mathrm{~cm}) \\
\end{array}$ \\
\hline \multirow[t]{6}{*}{$R T-Q M-01$} & 1 & 0.76 & - & \multirow[t]{6}{*}{$\overline{R T-Q M-04}$} & 1 & 0.51 & - \\
\hline & 2 & - & 0.13 & & 2 & - & 0.022 \\
\hline & 3 & - & -0.41 & & 3 & - & -0.099 \\
\hline & 4 & 0.20 & - & & 4 & 0.11 & - \\
\hline & 5 & - & - & & 5 & - & - \\
\hline & 6 & - & - & & 6 & - & - \\
\hline \multirow[t]{6}{*}{ RT $-Q M-02$} & 1 & -0.31 & - & \multirow[t]{6}{*}{$R T-Q M-05$} & 1 & -0.27 & - \\
\hline & 2 & - & -0.25 & & 2 & - & -0.11 \\
\hline & 3 & - & 0.91 & & 3 & - & 0.45 \\
\hline & 4 & -0.072 & - & & 4 & -0.057 & - \\
\hline & 5 & - & - & & 5 & $=$ & - \\
\hline & 6 & - & - & & 6 & $=$ & - \\
\hline \multirow[t]{6}{*}{$\mathrm{RT}-\mathrm{QM}-03$} & 1 & -0.037 & - & \multirow[t]{6}{*}{ RT $-Q M-06$} & 1 & -0.040 & - \\
\hline & 2 & - & 0.013 & & 2 & - & -0.022 \\
\hline & 3 & - & 0.053 & & 3 & - & 0.099 \\
\hline & 4 & -0.010 & - & & 4 & -0.0097 & - \\
\hline & 5 & - & - & & 5 & - & - \\
\hline & 6 & - & - & & 6 & - & - \\
\hline \multirow[t]{6}{*}{ Triplet } & 1 & 0.47 & - & \multirow{6}{*}{ RT $-Q M-07$} & 1 & 0.22 & - \\
\hline & 2 & - & -0.37 & & 2 & - & 0.028 \\
\hline & 3 & - & 0.45 & & 3 & - & -0.14 \\
\hline & 4 & 0.12 & - & & 4 & 0.15 & - \\
\hline & 5 & - & - & & 5 & - & - \\
\hline & 6 & - & - & & 6 & - & - \\
\hline \multirow[t]{6}{*}{ RT - BM-01 } & $\mathbf{I}$ & -0.47 & - & \multirow[t]{7}{*}{ RT -QM-08 } & 1 & -0.054 & - \\
\hline & 2 & - & -0.11 & & 2 & - & -0.0073 \\
\hline & 3 & - & 0.071 & & 3 & - & 0.046 \\
\hline & 4 & -0.15 & - & & 4 & -0.057 & - \\
\hline & 5 & 0.19 & - & & 5 & - & - \\
\hline & 6 & - & -0.61 & & 6 & - & - \\
\hline \multirow[t]{5}{*}{$\mathrm{RT}-\mathrm{BM}-02$} & 1 & -0.14 & - & & - & & \\
\hline & $\begin{array}{l}2 \\
3\end{array}$ & - & $\begin{array}{r}-0.029 \\
0.043\end{array}$ & \multirow{9}{*}{\multicolumn{4}{|c|}{ 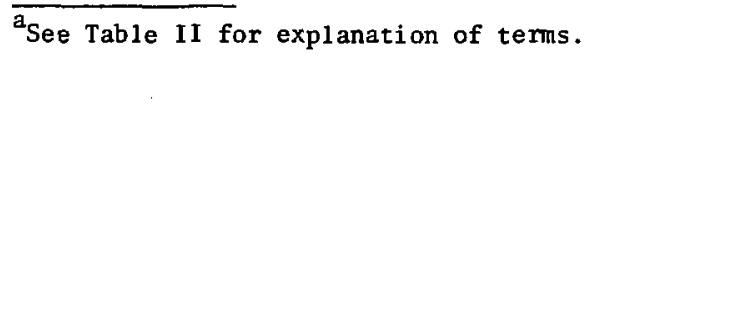 }} \\
\hline & 4 & -0.021 & $\begin{array}{c}0.040 \\
-\end{array}$ & & & & \\
\hline & 5 & 0.17 & - & & & & \\
\hline & 6 & - & -0.11 & & & & \\
\hline \multirow[t]{5}{*}{$\mathrm{RT}-\mathrm{BM}-03$} & 1 & 0.38 & - & & & & \\
\hline & 2 & - & -0.10 & & & & \\
\hline & 3 & - & -0.29 & & & & \\
\hline & 4 & 0.069 & - & & & & \\
\hline & $\begin{array}{l}5 \\
6\end{array}$ & 0.096 & $-0, \overline{30}$ & & & & \\
\hline
\end{tabular}

coincident with the exit reference point.

The remaining quadrupoles in the channel will be aligned along the channel axis following RT-BM-03 and with respect to its exit point.

2. Alignment. The entrance reference point is to be $1.251 \mathrm{~m} \pm 0.050 \mathrm{in}$. from the RT-BM-02 exit point and both entrance and exit reference points are to be within $\pm 0.050 \mathrm{in}$. of the channel axis in both the $x$ and $y$ directions. The reference plane is to be within \pm 0.050 in. of the bend plane at all points between the poles.
E. Alignment of the Last Five Quadrupoles (RT-QM04 through RT-QM-08

The entrance reference points of RT-QM-04 through RT-QM-08 are to be positioned along the channel axis at $1.000 \mathrm{~m}, 1.840 \mathrm{~m}, 2.680 \mathrm{~m}, 3.520 \mathrm{~m}$, and $4.360 \mathrm{~m}$ respectively from the $\mathrm{RT}-\mathrm{BM}-03$ exit point to within $\pm 0.050 \mathrm{in}$. Both the entrance and exit reference points of all five quadrupoles are to be within $\pm 0.050 \mathrm{in}$. of the channel axis in both the $x$ and $y$ directions. The quadrupole reference planes are to 

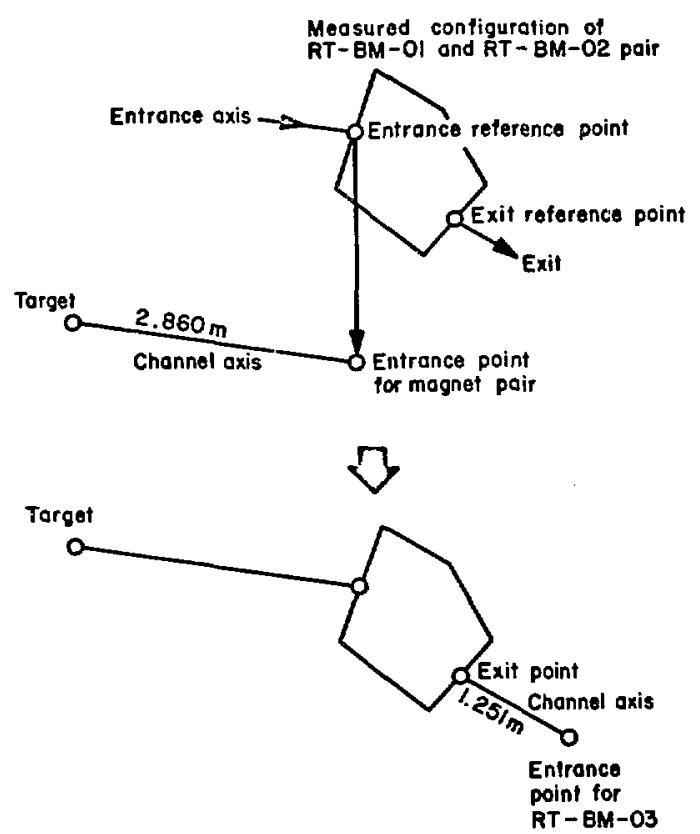

Fig. 2. Definition of channel axis and entrance and exit points associated with the magnet pair comprising RT-BM-01 and RT-BM-02.

be within $\pm 0.050 \mathrm{in}$. of the bend $\mathrm{plane}$ at all points within the bore of the magnets.

VI. DISCUSSION OF RESULTS OF SPECIFIED ALIGNMENT A. Significance of Stated Results

To first order a beam centroid shift is a linear function of the magnitude of misalignment of a magnet, and the centroid shift caused by any one magnet misalignment is independent of the misalignment of any other magnet. A uniform probability of misalignment of a magnet between two limits results in a uniform probability of the beam centroid shift being between two corresponding limits.

If a number of magnets are contributing to a centroid shift, in this way, the cumulative probability of a particular value of centroid shift follows a gaussian-like distribution. The mean-square value of the centroid shift due to misalignment of the $i^{\text {th }}$ magnet is just $\sigma_{i}^{2}=\left(\Delta x_{i}\right)^{2} / 3$, where $\Delta x_{i}$ is the magnitude of the worst case centroid shift arising from misalignment of that magnet. The cumulative value of the mean-square centroid shift for all magnets is then $\sigma^{2}=\sum\left(\Delta x_{1}\right)^{2} / 3$ where the summation is over all magnet $\frac{i}{s}$ or sets of magnets that 8

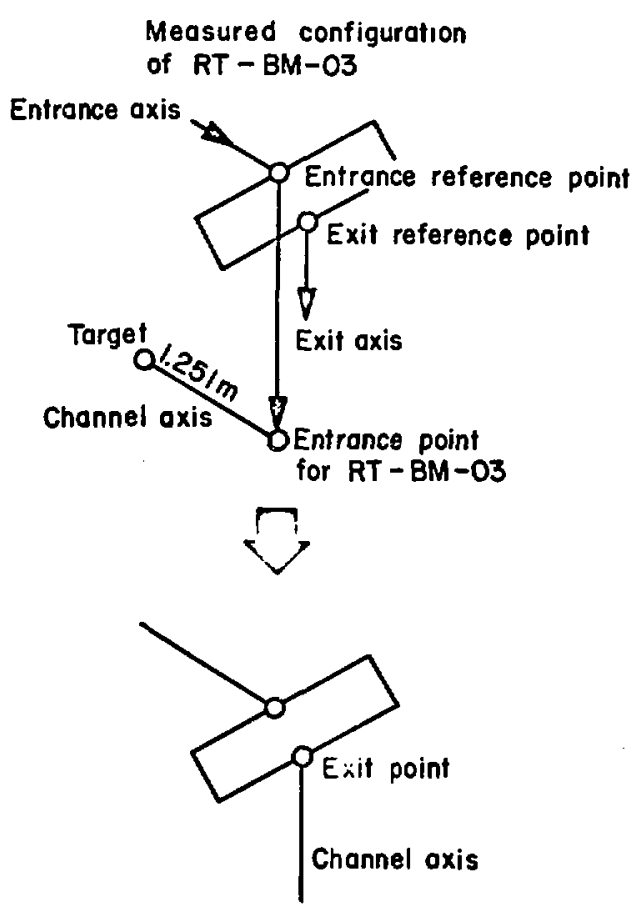

Fig. 3. Definition of channel axis and entrance and exit points associated with RT-BM-03.

are aligned as a unit.

In this case, the position of the centroid will be within $\pm 2 \sigma$ of the desired position with a probability of approximately $95 \%$.

B. Centroid Shifts at the Wedge Energy Degrader

Table $V$ shows that the rms value of the bend plane ( $x$ direction) centroid shift distribution is $0.08 \mathrm{~cm}$. This is negligible considering that the dispersion at this position is $0.6782 \% \mathrm{~cm} / \% \frac{\Delta \mathrm{P}}{\mathrm{P}_{\mathrm{O}}}$ of dispersion.

:he rms centroid shift in the transverse plane (y direction) is $0.30 \mathrm{~cm}$. This is also negligible.

Centroid shifts of the angular distribution are also small.

c. Beam Centroid Shifts at the Pion Irradiation Position

The centroid shifts at the end of channel as shown in Table $V$ are acceptable.

D. Beam Centroid Shifts within the Channel Magnets

Within the channel magnets, the beam envelope size is several centimeters smaller than the magnet aperture at most points along the channel. The beam 
centroid shifts within the magnets as shown in Table $v$ are calculated on the assumption that the worstcase beam centroid shift due to each magnet misalign ments is combined with the worst case shifts due to all other magnets. The resulting values of centroid shifts greatly overestimate the centroid shift at any one place along the channel but represents a conservative number for evaluating the significance of the shifts. The values shown are acceptable.

REFERENCES

1. Karl L. Brown and Sam K. Howry, "TRANSPORT/360. A Computer Program for Designing Charged

Particle Beam Transport Systems," Stanford Linear Accelerator report SLAC-91 (July 1970).
TABLE V

ROOT-MEAN-SQUARE CENTROID SHIFTS DUE TO USE OF ALIGNMENT TOLERANCES SPECIFIED IN SECTION $V$

Channel
Position
Wedge
End of channel
Inside magnets

$\mathrm{x}$ Centroid Shifts

(cm) (cm) (mr) (mr)

$\begin{array}{llll}0.08 & 0.30 & 2.4 & 1.4\end{array}$

$0.73 \quad 0.57$

$4.0 \quad 18.2$

$\mathrm{CM}: 241(50)$ 